\title{
Virtualizing National Broadband Access Infrastructure
}

\author{
Hassan Habibi Gharakheili and Vijay Sivaraman \\ School of Electrical Engineering and Telecommunications \\ University of New South Wales, Sydney, Australia \\ \{h.habibi, vijay\}@unsw.edu.au
}

\section{Categories and Subject Descriptors}

C.2.1 [Computer-Communication Networks]: Network Architecture and Design

\section{Keywords}

Software-defined Networking; Slicing; Capacity Planning

\section{MOTIVATION}

Governments world-wide are recognizing the importance of residential broadband in fueling economic and social growth of the nation. Unlike privately owned networks, such public networks will provide a wholesale platform on which retail service providers (RSPs) can compete to offer their services to consumers. This creates a unique opportunity to "virtualize" the wholesale access infrastructure (i.e. the fiber network) in a dynamic and agile way, allowing multiple retail service providers to share the infrastructure more efficiently, while giving users more freedom and choice in selecting their service mix. This poster presents our vision of how each party (user, wholesale infrastructure operator, and retail service provider) can benefit from this virtualization, how this virtualization can be achieved using software defined networking (SDN) techniques, and what some of the challenges in achieving this are.

\section{OPPORTUNITY}

Deploying fibre to every household is a large and expensive undertaking, with returns on investment often being over long periods of time and in indirect ways. That is why several governments, particularly in Asia-Pacific, either provide stimulus to industry to undertake FTTH deployments (Korea, Japan), or directly fund these from the public purse (Singapore, Malaysia, Australia). Of particular interest to the authors of this paper is the ongoing construction of a National Broadband Network (NBN) in Australia that aims to provide $100 \mathrm{Mbps}$ to over $93 \%$ of households in the country at an overall estimated cost of around $\$ 40$ billion. The archi-

Permission to make digital or hard copies of all or part of this work for personal or classroom use is granted without fee provided that copies are not made or distributed for profit or commercial advantage and that copies bear this notice and the full citation on the first page. Copyrights for components of this work owned by others than ACM must be honored. Abstracting with credit is permitted. To copy otherwise, or republish, to post on servers or to redistribute to lists, requires prior specific permission and/or a fee. Request permissions from permissions@acm.org.

CoNEXT Student Workshop'13, December 9, 2013, Santa Barbara, CA, USA.

Copyright 2013 ACM 978-1-4503-2575-2/13/12 ...\$15.00.

http://dx.doi.org/10.1145/2537148.2537152. tecture of such a public network is fundamentally different from a private network in several ways [6]: (a) it only offers wholesale connectivity to premises via layer-2 circuits, upon which retailers can offer their (layer-3) services to end-users, and (b) the infrastructure provides a level playing field for retailers to compete to offer their services to end-users.

The above architecture creates new and exciting opportunities that are not conceivable in a private (monopolistic) network. The public network, by definition, needs to promote sharing amongst retail operators, and hence virtualization (dynamic sharing) of the infrastructure can play a much more significant role. However, to the best of our knowledge, this opportunity has not been explored in sufficient depth for public networks, and we seek to address this gap in our research.

To appreciate the opportunities that virtualization [1] opens up, we note three important trends that are emerging: (a) Content providers (YouTube, NetFlix) that operate overthe-top are frustrated by the lack of innovation and service quality in current access network infrastructure, and would benefit from having direct access to their customers instead of relying on the (Telco) intermediary (this may provide a partial explanation for Google's large investment into building fibrehoods). A public fibre infrastructure should reduce the barrier for any entity to directly connect to its customers. (b) Household networks are becoming increasingly complex, both in terms of number of devices (media gateways, smart TVs, tablets, phones, PCs) and applications (video conferencing, VoIP, streaming, gaming), and users should not have to rely on one ISP to support these diverse requirements. A public fibre infrastructure should allow users to choose bestof-breed providers for each service. (c) Software Defined Networking is rapidly maturing as a technology that can achieve the virtualization of the network. Together, these trends provide compelling reasons to consider a more dominant role for virtualization in the public access infrastructure.

\section{BENEFITS OF VIRTUALIZATION}

Retail Service Provider (RSP): An RSP is required to provision its service through the public access infrastructure. Today's model uses static provisioning - for example the "NNI" (Fig. 1(a)) interconnect between the RSP and the NBN can be provisioned at $\$ 20$ per Mbps per month [5]. This static provisioning requires the RSP to do careful capacity planning based on forecast demand. The higher they provision the bandwidth, the more they pay, irrespective of actual load. If provisioned too low, however, temporary 

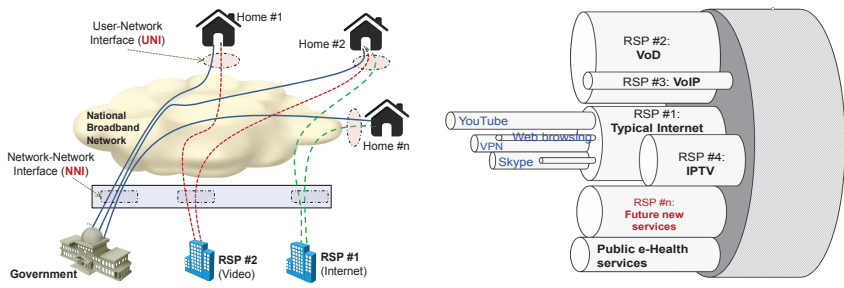

(a) Wholesale access topology (b) Virtualized access interface

Figure 1: Access Infrastructure

traffic spikes can result in poor experience for users, causing unhappiness. If the network infrastructure allowed dynamic partitioning, the RSPs could provision their bandwidth in a more elastic way, by modifying it on-the-fly as the aggregate user demand varies. This makes their cost scale with demand (i.e. revenue).

End-User: End-users have an increasing array of IPenabled devices in their houses that use a myriad set of services with diverse requirements - for example video streaming may require consistent bandwidth, real-time voice/video requires low latency, and gaming may require low loss. Further, as new services emerge, such as remote health, distance education, etc., users may want to avail of these services from specialised providers. Given these diverse needs, we believe the access infrastructure should reduce the barriers for specialist providers to offer niche services. A public fiber infrastructure should therefore provide agile, open and automated interfaces by which the network resource usage can be optimized [4].

Infrastructure Operator: Network virtualization facilitates more efficient use of resources for the public infrastructure [3], since capacity which is relinquished by one RSP can be used by another. Moreover, it is more competitive, as it allows specialised RSPs to offer niche services without taking on the whole bundle (Fig. 1(b)). This "unbundling" may also allow Content Providers to directly access customers without becoming a full-fledged ISP.

\section{VIRTUALIZATION APPROACH}

The concept of separating network infrastructure providers from network service providers has been deployed, under the name of "Open Access Networks" [2, 7]. However, this model does not envisage allowing a residential user to have multiple (specialized) network service providers. Sharing of network infrastructure amongst multiple operators has been proposed in [8], but challenges around dynamic sharing of resources amongst them have not been addressed. We believe the most suitable candidate technology for realizing this is software defined networking (SDN), due to its open and centralised nature, and ease of integration with APIs that can be exposed to external parties.

The architecture could broadly be as follows (see Fig. 1(a)): the network infrastructure operator provisions tunnels (e.g. using q-in-q tagging) from the NNI (where RSPs connect to it) to end-user premises. Traffic to/from end-user devices is mapped to the appropriate RSP tunnel based on policy that could be associated with a device or an application (for example, the TV is mapped to the video RSP tunnel and the glucose monitor is mapped to the health RSP tunnel, or the YouTube application traffic is mapped to Google as the RSP). These tunnels are provisioned with a low base bandwidth, and the network infrastructure operator pro-

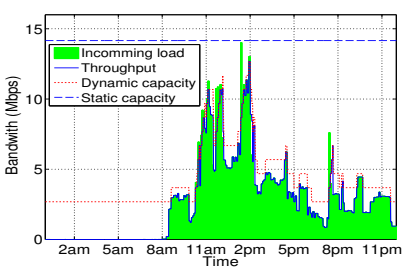

(a) Dynamic capacity

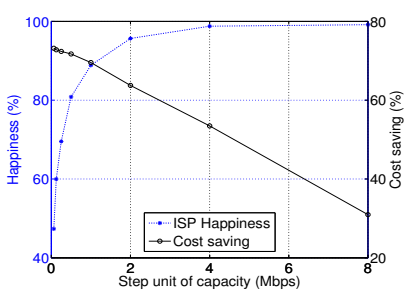

(b) Measure of performance
Figure 2: Dynamic allocation of capacity

vides APIs for the RSP to up/down-scale their bandwidth on-demand. So for example when the user starts watching $\mathrm{TV}$, the RSP serving the content can choose to augment user QoE by dynamically calling the API to dilate bandwidth for this stream at the UNI (and if needed also at the NNI). This bandwidth can be relinquished by the RSP when the user abandons the video, and adjusted as needed (for example if the user wants to upgrade to HD video). This fine-grained management, enabled by the dynamic API, gives the RSP elasticity to control their resource usage (and cost) in line with their business priorities.

\section{PRELIMINARY RESULTS}

To demonstrate that our model allows an RSP to manage their costs while maintaining user QoE at a desired level, we apply it in simulation to a real trace data taken from our university campus WiFi network. To make our study tractable, we chose to focus on just one building of 6 levels which houses $30 \mathrm{WiFi}$ access points (APs), and can be thought to represent a multi-storey dwelling unit comprising many households. Our traffic trace, taken over 24 hours, had approximately 8500 user sessions. We wrote a native simulation that takes sessions arrival, throughput and duration from the trace file as input, and maps each user device (MAC address) randomly to one of 4 different RSPs, assumed to have market-share of $10 \%, 20 \%, 30 \%$ and $40 \%$ respectively. Note that an AP may serve client sessions from different RSPs, allowing a home to have multiple RSPs.

Every RSP is initially provisioned a minimum "base" capacity that is proportional to average daily demand. Periodically (every 1 minute), the RSP can call the API to dilate or shrink bandwidth available to it from the infrastructure in steps of $1 \mathrm{Mbps}$, thereby managing its costs. The fluctuation in bandwidth demanded by the largest RSP is shown in Fig. 2(a), while the dashed flat line shows the static provisioning based on peak load. Bandwidth fluctuations can degrade performance for user sessions, since the RSP's bandwidth increments can lag session traffic rate requirements - this is captured by the "happiness" metric that is measured as a percentage of session traffic that does not get its required bandwidth. Fig. 2(b) shows that allocating bandwidth in larger steps (x-axis) improves user happiness but reduces cost savings compared to static provisioning.

\section{CONCLUSIONS}

We have considered public network infrastructure that can be shared by many retail network service providers (RSPs), and shown how APIs powered by SDN can enable dynamic partitioning of network resources. We have argued that this benefits all parties: RSP can scale costs with revenues, users can benefit from improved QoE, and the infrastructure can achieve efficient utilization. 


\section{REFERENCES}

[1] J. Carapinha and J. Jiménez. Network virtualization: a view from the bottom. In Proc. ACM SIGCOMM Workshop on Virtualized Infastructure Systems and Architectures, Aug. 2009.

[2] M. Forzati, C. P. Larsen, and C. Mattsson. Open Access Networks, the Swedish Experience. In Proc. ICTON, July 2010.

[3] C.-Y. Hong, S. Kandula, R. Mahajan, M. Zhang, V. Gill, M. Nanduri, and R. Wattenhofer. Achieving high utilization with software-driven WAN. In Proc. ACM SIGCOMM, Aug. 2013.

[4] S. Hubbard. Implementing the Innovative Edge for Cloud-Based Services. White Paper, Juniper Networks, Nov. 2012.
[5] NBN Co. Wholesale access service: Product and pricing overview for access seekers. http://www.nbnco.com.au, Dec. 2011.

[6] V. Sivaraman, C. Russell, I. B. Collings, and A. Radford. Architecting a national optical fiber open-access network: The australian challenge. Network, IEEE, 26(4):4-10, 2012.

[7] P. Sköldström, A. Gavler, and V. Nordell. Virtualizing Open Access Networks. In Proc. SNCNW, June 2011.

[8] Y. Yiakoumis, K. Yap, S. Katti, G. Parulkar, and N. McKeown. Slicing Home Networks. In Proc. ACM SIGCOMM HomeNets Workshop, Aug. 2011. 